\title{
STATISTICAL STUDY OF FREE MAGNETIC ENERGY AND FLARE PRODUCTIVITY OF SOLAR ACTIVE REGIONS
}

\author{
J.T. Su ${ }^{1,2,3}$, J. Jing ${ }^{1}$, S. Wang ${ }^{1,2}$, T. Wiegelmann ${ }^{4}$ and H.M. Wang ${ }^{1}$ \\ 1. Space Weather Research Laboratory, New Jersey Institute of Technology, University \\ Heights, Newark, NJ 07102-1982, USA \\ 2. Key Laboratory of Solar Activity, National Astronomical Observatories, Chinese \\ Academy of Sciences, Beijing 100012, China \\ 3. State Key Laboratory of Space Weather, Chinese Academy of Sciences, Beijing 100190 \\ 4. Max-Planck-Institut fur Sonnensystemforschung, Max-Planck-Strasse 2, 37191 \\ Katlenburg-Lindau, Germany \\ sjt@bao.ac.cn
}

Received —

Not to appear in Nonlearned J., 45. 


\begin{abstract}
Photospheric vector magnetograms from Helioseismic and Magnetic Imager on board the Solar Dynamic Observatory are utilized as the boundary conditions to extrapolate both non-linear force-free and potential magnetic fields in solar corona. Based on the extrapolations, we are able to determine the free magnetic energy (FME) stored in active regions (ARs). Over 3000 vector magnetograms in 61 ARs were analyzed. We compare FME with ARs' flare index (FI) and find that there is a weak correlation $(<60 \%)$ between FME and FI. FME shows slightly improved flare predictability relative to total unsigned magnetic flux of ARs in the following two aspects: (1) the flare productivity predicted by FME is higher than that predicted by magnetic flux and (2) the correlation between FI and FME is higher than that between FI and magnetic flux. However, this improvement is not significant enough to make a substantial difference in timeaccumulated FI, rather than individual flare, predictions.
\end{abstract}

Subject headings: Sun: activity — Sun: flares — Sun: magnetic fields 


\section{INTRODUCTION}

Solar flares are sudden brightening observed over the Sun's surface or above the solar limb. The energy release in a flare varies from $10^{29}$ ergs in a sub-flare to $10^{32}$ ergs in a typical large event (Priest 1984). It could be up to $10^{34}$ ergs in the largest events such as the one on 4 November 2003 (Kane et al. 2004). The source of its energy is generally believed coming from the magnetic energy, $E=\frac{1}{8 \pi} \int B(\mathbf{r}, t)^{2} d V$, stored primarily in active regions (ARs). This energy has a minimum when the coronal electric current vanishes (potential field configuration), and increases in value when the field becomes twisted or open (Aly 1990). The development of flaring conditions does not depend on the total magnetic flux, but depends on the availability of free magnetic energy (FME) and the triggering processes to release it (Low 1982). The FME in magnetic field is the amount of magnetic energy in excess of the minimum energy attributed to the potential field $\mathbf{B}(\mathbf{r}, t)^{p}$ (Sturrock 1967). It can be roughly estimated from vector magnetograph data on the photosphere with the Virial expression (Molodensky 1974)

$$
E_{\text {free }}=\frac{1}{4 \pi} \iint B_{z}\left[x\left(B_{x}-B_{x}^{p}\right)+y\left(B_{y}-B_{y}^{p}\right)\right] d x d y
$$

where $\mathbf{B}(\mathbf{r}, t)$ is assumed to be force-free and vanishes when $\left(x^{2}+y^{2}+z^{2}\right)^{1 / 2} \rightarrow \infty$. The formula can also be extended with the Virial theorem to the case in which the field is not force-free but in a static equilibrium with plasma pressure and gravity (Low 1982). In the latter case, the total energy of the system contains not only the magnetic energy, but also thermal and gravitational energy. In general, when using Eq. (1) to estimate FME, it is hard to estimate errors that would be introduced in the calculations, as the fields are neglected in the lateral and upper boundaries.

At present, exact measurements of the three dimensional (3-D) fields are not yet available and vector magnetic fields are only measured in the photospheric level. Methods

using magnetically sensitive coronal spectral lines (Judge et al. 2013) or coronal seismology 
(Van Doorsselaere et al. 2007) have been developed. These methods, however, do not provide a quantitative 3-D structure of the coronal fields. With the observed photospheric vector magnetic fields, there are several ways to obtain the 3 -D magnetic field in the corona. One of them is the data-driven magnetohydrodynamics (MHD) modeling (Wu et al. 2006). This approach, although more computationally intensive and less routinely used, gives impressive results in recent studies (Jiang et al. 2012; Fan et al. 2011). Another useful approach is the well-known nonlinear force-free (NLFF) field extrapolation, using the vector photospheric magnetograms as the boundary condition (Wiegelmann 2004; Schrijver et al. 2008; De Rosa et al. 2009). With a NLFF field, FME may be evaluated by the expression

$$
E_{1 \text { free }}=\frac{1}{8 \pi} \int\left(B^{2}-B^{p 2}\right) d V,
$$

which has been used in many recent studies (e.g., Jing et al. 2010; Gilchrist et al. 2012). Similarly, there is another expression to evaluate FME

$$
E_{2 \text { free }}=\frac{1}{8 \pi} \int\left(\mathbf{B}-\mathbf{B}^{p}\right)^{2} d V
$$

where $\mathbf{B}-\mathbf{B}^{p}$ is called the source field to describe the nonpotentiality of the magnetic field (Hagyard et al. 1981; Wang et al. 1996). In general, Eq. (2) gives the energy difference between the NLFF field $\mathbf{B}$ and the respective potential field $\mathbf{B}^{p}$, while Eq. (3) gives the total energy of the non-potential field $\mathbf{B}-\mathbf{B}^{p}$. Their difference, over the entire volume $V$, amounts to zero, given the well-known decomposition of the non-potential field $\mathbf{B}$ into poloidal $\mathbf{B}^{p}$ and toroidal $\mathbf{B}-\mathbf{B}^{p}$ components. Using the above two equations, we may define a mean value to estimate FME, namely

$$
E_{3 \text { free }}=\frac{1}{2}\left(E_{1 \text { free }}+E_{2 \text { free }}\right)
$$

with uncertainty

$$
\Delta E_{\text {free }}=\frac{1}{4 \pi} \int\left|\mathbf{B}^{p} \cdot\left(\mathbf{B}^{p}-\mathbf{B}\right)\right| d V,
$$


which is derived from Eq.(2)-Eq.(3). The magnetic field measurements and errors in extrapolation contribute to this uncertainty. In addition, $E_{\text {free }}$ determined with Eq.(4) is regarded as the upper limit of the energy that is available to power flares/CMEs because not all of the FME mentioned above is free. Constrained by the conservation of total magnetic helicity, the lowest energy state of ARs is actually linear force free (LFF) field instead of potential field (Woltjer 1958; Taylor 1974, 1976). In this case, the FME should be estimated by $E_{\text {free }}=E-E_{l f f}$, the difference between the total energy and the energy of a LFF field. However, LFF fields are unrealistic for the open upper halfspace, because the energy is unbounded (see Seehafer 1978). Thus, one often finds $E_{\text {free }}$ from the difference $E-E_{l f f}$ being negative.

In the past three decades, as the diagnosis of magnetic fields in the chromosphere and corona is difficult, various surface nonpotentiality indices of the solar ARs have been developed to predict flares (see Leka \& Barnes 2003; Yang et al. 2012), such as magnetic shear angle (e.g., Hagyard et al. 1984; Ambastha et al. 1993; Wang et al. 1994; Li et al. 2000; Tian et al. 2002), electric current (e.g., Canfield et al.|1993; Leka 1999; Wang et al. 1996; Zhang 2001), current helicity (e.g., Seehafer 1990; Bao et al. 1999), subsurface kinetic helicity (Gao et al. 2012, 2014), horizontal magnetic gradient (e.g., McIntosh 1990; Zirin \& Wang 1993; Zhang et al. 1994; Tian et al. 2002; Cui et al. 2006), length of the high-gradient and high-sheared polarity inversion line (Falconer et al. 2003; Schrijver et al. 2005; Cui et al. 2006; Jing et al. 2006), effective distance between two opposite magnetic polarities in one AR (Chumak et al. 2004; Guo et al. 2006) and photospheric excess energy (Wang et al. 1996; Metcalf et al. 1995; Moon et al. 2002; Leka \& Barnes 2003). Compared with those photospheric nonpotentiality indices, FME derived from 3-D coronal magnetic configuration over ARs seems to be a more direct physical parameter related to the available energy to power flares. Based on 75 AR's magnetograms, Jing et al. (2010) conducted a pilot study of the statistical correlation between FME derived from 3-D NLFF 
fields and flare index (FI) (Antalova 1996; Abramenko 2005). They found a positive correlation between the FME and flare productivity of solar ARs. In this study, we revisit this issue by using a much more extended sample of AR vector magnetograms obtained with SDO/HMI. We study both FME and total unsigned magnetic flux $(\Phi)$ of ARs in order to compare their respective abilities to predict flare productivity. In addition, we explore the statistical correlation between FI and other magnetic parameters: the total unsigned electric current $I_{t}=\int\left|J_{z}\right| d s=\frac{1}{4 \pi} \int\left|\partial B_{y} / \partial x-\partial B_{x} / \partial y\right| d s$ (Canfield et al. 1993; Leka 1999), the averaged twisted measure $\alpha_{a v}=\sum J_{z}(x, y) \operatorname{sign}\left[B_{z}(x, y)\right] / \sum\left|B_{z}(x, y)\right|$ (Hagino \& Sakurai 2004), and the proxy of photospheric excess energy $E_{p e}=\frac{1}{8 \pi} \int\left(\mathbf{B}-\mathbf{B}^{p}\right)^{2} d s$. Table 1 lists all the parameters investigated in this paper.

\section{DATA REDUCTION}

The data sample includes 6261 photospheric vector magnetograms covering 61 ARs. The center locations of these magnetograms are within 45-degree from the disk center, and their disk passage is in the period of May 2010 through April 2013 as listed in Table 2. The magntograms are obtained with the Helioseismic and Magnetic Imager (HMI) on board the Solar Dynamics Observatory (SDO). We use the SHARP (Spaceweather HMI Active Region Patches) data series (Turmon et al. 2010) which are re-mapped in Cartesian coordinates using Cylindrical Equal Area (CEA) projection. The 180-degree ambiguity in the transverse field has been resolved with Metcalf's Minimum Energy method (Leka et al. 2009). The resolution of the original magnetogram data is about $0 .^{\prime \prime} 5$ pixel $^{-1}$. To speed up extrapolation, we lower the resolution and rebin them to $3^{\prime \prime}$ pixel $^{-1}$. We preprocess the photospheric magnetograms using a method devised by Wiegelmann, Inhester \& Sakurai (2006). NLFF fields and potential fields are then extrapolated from the preprocessed photospheric boundary with the weighted optimization (Wiegelmann 2004) and FFT 
(Seehafer 1978) methods, respectively.

Note that we do not include smoothing in the data preprocessing. To investigate how the results vary due to the smoothing, we carry out some testing extrapolations. Two sets of vector magnetograms are used as the bottom boundary of the NLFF field extrapolation: the magnetograms smoothed over a $3^{\prime \prime} \times 3^{\prime \prime}$ area, and the same magnetograms without smoothing. The magnetograms used here were taken in AR 11158 spanning from 00:00 UT of 2011 February 12 to 15:48 UT of February 15 with a cadence of 12 min. Figure 1(a) shows $E_{3 f r e e}^{s}$ (with smoothing) versus $E_{3 f r e e}^{n s}$ (without smoothing). The blue line is a linear fitting to the data. The smoothed results seem to diverge for $E_{3 f r e e}^{s}>4 \times 10^{32}$ ergs, but below this limit they give very similar FME values to those obtained without smoothing. Even so, the rank correlation coefficient (RCC), which assesses how well the relationship between two variables and can be described using a monotonic function, is very high up to $93 \%$. We give the correlation's confidence level defined as $\left(1-P_{s}\right) \times 100 \%$ shown in parentheses in the bottom right corner of panels (a) and (c), where $P_{s}$ is the probability (binomial distribution) that the observed correlation would occur by random chance. It is calculated by the routine erfcc described in section 14.6 of Numerical Recipes (Press et al. 1992). We also evaluate the variation of FMEs from the magnetograms in different spatial resolution. The data of AR 11158 at 19:48:00 UT on 2011 February 13 is used. The obtained values are $1.84 \times 10^{32}, 1.89 \times 10^{32}$ and $1.64 \times 10^{32}$ ergs, corresponding to the spatial resolutions of, $1.5^{\prime \prime}, 2.0^{\prime \prime}$ and $3.0^{\prime \prime}$, respectively. Therefore, FME does not change significantly with different resolutions, so it would not affect our conclusion.

Now we consider the magnetic flux imbalance in our data sample. It may be evaluated by the ratio of the net flux to the total unsigned one in the field of view, $r_{i m}=\left|\sum B_{z} d s\right| / \sum\left|B_{z}\right| d s$. A histogram of $r_{i m}$ for all the data is shown in Figure 1(b). It is found that except for some data with a very large $r_{i m}$ (e.g. 0.6), most of the data 
$(>76 \%)$ shows a value of $r_{i m}$ less than 0.2 .

After these procedures, we obtain all the extrapolated 3-D fields based on 6261 vector

magnetograms. $E_{1 \text { free }}$ and $E_{2 \text { free }}$ from Eqs. (2) and (3) are then determined subsequently. Figure $1(\mathrm{c})$ shows a scatter plot of $E_{1 \text { free }}$ versus $E_{2 \text { free }}$ for all the data. Their correlation is very high, up to $\sim 93 \%$. A linear fit (blue line) shows that $E_{2 \text { free }}$ is greater than $E_{1 \text { free }}$ with a factor $\sim 1.1$. Their difference (see Eq. (5)) can be used to evaluate the uncertainty of $E_{3 \text { free. }}$ For our case, however, the relative error may be more helpful. Therefore, we define a relative one of $E_{3 f r e e}$, namely $r_{u n}=\Delta E_{\text {free }} / E_{3 \text { free }}$, whose histogram is shown in Figure $1(\mathrm{~d})$. We find that $\sim 71 \%$ of FMEs is with $r_{u n} \leqslant 0.3$. To further ensure the reliability of the analysis, we exclude both samples, those with the flux imbalance $r_{i m}>0.2$ and those with the relative FME uncertainty $r_{u n}>0.3$. Finally, the remained sample of 3226 vector magnetograms are used for further analysis.

\section{ANALYSIS AND RESULTS}

\subsection{DEFINITION OF FLARE INDEX}

Solar flares are conventionally classified as X, M, C, or B according to their peak soft X-ray (SXR) flux in the wavelength range 1 to 8 Angstroms, as measured by the Geostationary Operational Environmental Satellite (GOES) and recorded in the NOAA Space Environment Center's solar event reports. The peak flux of X-, M-, C- and B-class flares is of $10^{-4}, 10^{-5}, 10^{-6}$ and $10^{-7} \mathrm{~W} \mathrm{~m}^{-2}$ magnitude order, respectively. If given the beginning and ending time of a time window, then the dimensionless FI in this interval $\tau$ (usually measured in days) is defined as

$$
F I=\left(100 \times \sum_{\tau} I_{X}+10 \times \sum_{\tau} I_{M}+1 \times \sum_{\tau} I_{C}+0.1 \times \sum_{\tau} I_{B}\right) / I_{C 1.0}
$$


where $I_{X}, I_{M}, I_{C}$, and $I_{B}$ are the SXR peak flux of X-, M-, C-, and B-class flares, respectively, produced by one AR within $\tau$-days. This definition measures a timeaccumulated flare production, which is somehow different from the time-averaged flare production used in Antalova (1996) and Abramenko (2005). When we study the correlation between FME and flare production in a fixed time period, there is no difference between the time-accumulated FI used here and the time-averaged FI used in previous studies. However, they are different when we study the frequency distributions and the power-law indices of flare production (Subsection 3.2). Thereafter, the abbreviation of FI in this paper refers to the time-accumulated flare index. Moreover, to characterize the ARs with certain flare magnitudes, we set a series of thresholds of FI to study their correlations with the magnetic parameters of ARs. For example, if we investigate the $\geq \mathrm{C} 1.0, \geq \mathrm{M} 1.0$ and $\geq \mathrm{M} 5.0$ flare productions, then will set $F I \geq 1,10$ and 50, respectively. Accordingly, $F I \geqslant 0$ denotes all the ARs' data satisfying this condition, no matter whether the ARs are flare productive or not.

\subsection{POWER-LAW DISTRIBUTION OF FME AND FI}

Statistical analyses show that most of the frequency distributions of parameters related to solar activities can be characterized by power-law distributions (Aschwanden 2011), which reflects an underlying power law in the distribution of energy release (Akabane 1956; Hudson 1991; Wheatland 2000). We also find such a distribution in the histograms of FME, FI and magnetic flux shown in Figure 2. The histograms use all the data identified by $F I \geqslant 0$. We apply chi-square $\left(\chi^{2}\right)$ test to evaluate whether they obey power-law distribution $N(S) \sim S^{-\alpha}$ or not, where $S$ refers to $E_{3 \text { free }}, F I$ and $\Phi$, and $\alpha$ is the power-law index. The FME and FI data are divided into 10 bins, and those of magnetic flux, 15 bins. Figure $2(\mathrm{~b})$ shows that one of the bins in the histogram $(350 \sim 600)$ of $F I_{1-d a y}$ has the 
sample size $N<5$, so the $\chi^{2}$-test cannot be applied to this histogram. The test to the other histograms shows that the values of $\alpha$ are $\sim 2$ and 1.8 for the FME and magnetic flux distribution, respectively, and $\sim 1.5$ for FI in the time window of 2 - or 3-days, which are

comparable to the index of total energy release in soft X-ray (1.5-1.6), and that of the peak flux (1.7) (Song et al. 2012).

Table 3 lists the values of $\alpha$ when the thresholds of $F I$ are set as $F I \geqslant 0,1,10$ and 50 in the time windows $\tau=1-3$ days. The power-law index $1.4-1.5$ of $F I$ does not change much with the increasing thresholds, whereas that of $E_{3 \text { free }}$ decreases systematically from $\sim 2$ to 1.5. The index of magntic flux also shows a decrease, but with a large fluctuation. Generally, in most cases the difference between the power-law indices, of FI, FME, and the flux is insignificant, that is within measurement/fitting errors.

\subsection{FLARING PRODUCTIVITY AT DIFFERENT THRESHOLD OF FI}

We set 5 thresholds for FI, namely $F I \geq 0,1,10,50$ and 100 . The histograms of $E_{3 \text { free }}$ and $\Phi$ under these thresholds are shown in the first row of Figures 3 and 4, respectively. We here introduce the definition of flare productivity $P(X)=S_{a}(X) / S_{t}(X)$ (Cui et al. 2006), where $X$ is of one measure describing magnetic properties, $S_{a}(X)$ and $S_{t}(X)$ are the number of events (their flaring activity independent of strength) and the total samples, respectively. It denotes flaring productivity of the ARs under certain properties of nonpotentiality and magnetic flux. With this definition, $P\left(E_{3 f r e e}\right)$ and $P(\Phi)$ are obtained at different thresholds of FI, as illustrated in the second row of Figure 3 and 4, respectively. In these panels, the black solid lines are the Boltzmann sigmoidal fittings (Cui et al. 2006) to the curves of flare productivity. In the top panels of Figure 3, we notice that the data show discontinuity in the energy range of $8.5-12.3 \times 10^{32}$ ergs with a bump appearing at the position of $12.3 \times 10^{32}$ ergs. We thus plot a dotted-line crossing the blue-line bump ( $\geq$ M1.0 flares) 
in the first panel of Figure 3, which roughly corresponds to the sample number of 50 . Therefore, in order to guarantee statistical significance, we do not fit the flare productivity when $S_{t}(X)$ is less than 50 .

We attempt to further illustrate the flare productivity predicted by $E_{3 \text { free }}$ and $\Phi$ with a specific case. For instance, when the FME is taken at $5.0 \times 10^{32} \mathrm{ergs}$, roughly corresponding to the magnetic flux at $3.0 \times 10^{22} \mathrm{Mx}$ (see Figure $7(\mathrm{a})$ ), the corresponding flare productivity within the time windows of $\tau=1-3$ days can be obtained as listed in Table 4. Generally, $P$ declines rapidly with increasing threshold and rises steadily with the increase of the time window. Moreover, $P$ is higher as predicted by $E_{3 f r e e}$ in each time window at a given threshold, suggesting that this parameter can give a slightly better prediction for FI than $\Phi$. In addition, we cannot give the productivity when $E_{3 \text { free }}>8 \times 10^{32}$ ergs, as the sample size is inadequate $(N<50)$. It indicates that more $\mathrm{AR}$ samples that produce large flares (>M5.0) are needed to supplement this study to make a more accurate flare forecasting for larger events.

\subsection{CORRELATION OF FI WITH FME/MAGNETIC FLUX}

Figure 5 shows the scatter plots of $E_{3 \text { free }}$ versus $F I$ (top) and $\Phi$ versus $F I$ (bottom) for all the data $(F I \geqslant 0)$, respectively. In spite of the fact that the data are widely scattered, the results still reveal a weak positive correlation between the quantities. For instance, in $\tau=2$-days the RCC between $E_{3 \text { free }}$ and $F I$ is $57 \%$ and the RCC between $\Phi$ and FI 55\%. Generally, the correlations of $F I$ with $E_{3 \text { free }}$ and $\Phi$ are nearly equivalent in the selected time windows, which are comparable to, but less than, the $65 \%$ correlation between eruptive-flare production and AR sigmoidality as well as size (Canfield et al. 1999).

Furthermore, Figure 6 shows that the RCC varies with the thresholds of FI (panels (a) 
and (b)), FME (panel (c)) and magnetic flux (panel (d)). For convenience, we define here the threshold of FI as TFI. The relationship between them, e.g. $F I \geq 0,1, \ldots$ is equivalent to $T F I=0,1, \ldots$. Likewise, the threshold of FME is defined as TFME and that of magnetic flux as TMF. In the figure, the maximum of $T F I$ is set to be 50 (at this threshold $N=528$ ), that of $T F M E$ is $1.3 \times 10^{33} \mathrm{ergs}(N=50)$, and that of $T M F$ is $5.0 \times 10^{22} \mathrm{Mx}(N=106)$. Panels (a) and (b) respectively show the RCC between FME and FI and RCC between magnetic flux and FI as a function of TFI. Both RCCs change with a very similar trend: declining first when $T F I<10$, then rising at larger threshold $(10<T F I<26)$, an finally keeping nearly unchanged when $T F I>26$. Note that the variation of the RCCs in the range of $10<T F I<26$ is opposite to that of $T F I<10$, increasing with the decreasing time window. A similar feature can be found in Figure 6(d) for the correlation between $\Phi$ and $F I$, and the turnover point occurs at $\sim T M F=1.9 \times 10^{22} \mathrm{Mx}$. In Figure 6(c) for the correlation between $E_{3 \text { free }}$ and FI, the RCCs exhibit a large fluctuation with TFME. Those in the 2- and 3-day time windows change similarly in magnitude, but are all greater than that in the 1-day time window. A remarkable feature is that the RCCs decrease first when the threshold is less than $\sim 2.9 \times 10^{32}$ ergs, while then increase when it is greater than $\sim 6.5 \times 10^{32}$ ergs. However, in Figure $6(\mathrm{~d})$ the RCCs only show a monotonically decreasing trend in the entire range of $T M F$. This reflects that ARs with the larger FME, rather magnetic flux, are more favorable to produce flares (Low 1982).

Figure 7 shows the scatter plots of $\Phi$ versus $E_{3 f r e e}(\operatorname{panel}(\mathrm{a})), I_{t}$ (panel (b)), $E_{p e}$ (panel (c)) and $\alpha_{a v}$ (panel (d)) for all the data $(F I \geqslant 0)$, respectively. Except $\alpha_{a v}$, the correlations of $\Phi$ with the others are very high, more than $90 \%$. We note that there are likely two slopes in the $E_{3 \text { free }}-\Phi$ plot with the turning point occurs at $\sim \Phi=3 \times 10^{21} \mathrm{Mx}$. Similarly, we can find this feature in Figure 5(a) of Jing et al. (2010). Finally, we list the RCCs of $F I / \Phi$ with all the used measures in Table 5. Generally, the RCCs associated with FI are relatively weak $(\leq 65 \%)$, and those with $\Phi$ are very strong $(\geqslant 90 \%)$ except the measure of 
twist. This confirms reports that there is no discernible correlation between the magnetic twist measure and magnetic flux (Falconer et al. 2006).

\section{SUMMARY AND DISCUSSION}

In this paper, based on a data sample of 3226 vector magnetograms in 61 active regions, we present the frequency distributions of free magnetic energy (FME) (i.e., $E_{3 \text { free }}$ defined in Eq. (4)), flare index (FI), and total unsigned magnetic flux $(\Phi)$. We also analyze the potential of $E_{3 \text { free }}$ and $\Phi$ as flare predictors and examine the magnitude scaling correlation between FI and several magnetic measures such as $E_{3 \text { free }}, \Phi$, total unsigned electric current $I_{t}$, proxy of photospheric free magnetic energy $E_{p e}$ and averaged magnetic twist measure $\alpha_{a v}$.

It is found that the frequency functions of FME, FI and magnetic flux all exhibit a power-law distribution. The index of $E_{3 \text { free }}$ (see Table 3), which is steeper than that of $F I(\Phi)$, varies from the maximum 2.0 to $\sim 1.5$ with the threshold of $F I$. The difference between the power-law indices, of FI, FME, and the flux is insignificant. We also find that $E_{3 \text { free }}$ shows an improved flare predictability (demonstrated in Table 4) in comparison with $\Phi$. However, in terms of the magnitude scaling correlation between $F I_{\tau-\text { day }}$ and $E_{3 \text { free }}, \Phi$, $I_{t}, E_{p e}$ and $\alpha_{a v}$ (see Table 5) based on all the used samples, the magnetic measure of FME shows no improvement for flare predictability. This result is consistent with the previous study of Jing et al. (2010). We then set a series of thresholds for $F I, E_{3 \text { free }}$ and $\Phi$ to study how the above correlations vary with these thresholds. It is found that the correlation between $\Phi$ and FI shows a general decreasing trend with an increasing threshold of flux, while that between $E_{3 \text { free }}$ and FI increases when the threshold of FME is greater than $6.5 \times 10^{32}$ ergs. This suggests that ARs with the larger FME rather magnetic flux are more favorable to produce flares (Low 1982$)$. 
Generally speaking, despite the fact that $E_{3 \text { free }}$ is one of the most direct measures of the available energy in a 3-D magnetic field, our large-sample study shows that its correlation with $F I_{\tau-d a y}$ is still weak $(<60 \%)$. Jing et al. (2010) gave a detailed discussion about the cause of the lack of satisfactory results, such as the quality of the extrapolated NLFF fields which is influenced by a number of inadequacies of the boundary data, uncertainties in the data reduction, etc. In the present study, we first evaluate the flux imbalance in our chosen sample as shown in Figure 2(b) and find that most of the data $(>76 \%)$ are of with $r_{i m}<0.2$. We then evaluate the uncertainty in the determined FME and find $\sim 71 \%$ of the sample with $r_{u n}<0.3$ as shown in Figure 1(d). To ensure the reliability of the analysis, these two types of data are excluded from the original sample.

We also evaluated the energy in excess of the LFF component $E_{\text {free }}=E-E_{l f f}$ with the averaged twisted measure $\alpha_{a v}$ (Hagino \& Sakurai 2004), and found $E_{\text {free }}$ of nearly half of all the data is negative. These negative values hamper us to give a further analysis. One way to get around this problem is to find the relative magnetic helicity from the extrapolated NLFF field and infer the constant $\alpha$-value corresponding to this helicity, then further calculate a LFF field and its corresponding $E_{l f f}$. However, this is beyond the scope of the current paper.

The authors thank the anonymous referee for helpful suggestions and comments on the manuscript, which significantly improve the work. This work is supported by the Grants: National Basic Research Program of China under grant 2011CB8114001, the Specialized Research Fund for State Key Laboratories, the Key Laboratory of Solar Activity of CAS (KLSA201313), 11373040, KJCX2-EW-T07, 11178005, 11221063 and 11203036. JJ and HW are supported by US NASA grant NNX11AQ55G and NSF grants AGS 1153226, AGS 1153424 and AGS 1250374. 


\section{REFERENCES}

Abramenko, V.I. 2005, ApJ, 629, 1141

Akabane, K. 1956, PASJ, 8, 173

Aly, J.J. 1990, Physics of Fluids B, 2, 1928

Ambastha, A., Hagyard, M. J., \& West, E. A. 1993, Sol. Phys., 148, 277

Antalova, A. 1996, Contributions of the Astronomical Observatory Skalnate Pleso, 26, 98

Aschwanden, M.J. 2011, Self-Organized Criticality in Astrophysics, by Markus J. Aschwanden. Springer-Praxis, Berlin ISBN 978-3-642-15000-5, 416p.

Bao, S.D., Zhang, H.Q., Ai, G.X., \& Zhang, M. 1999, A\&AS, 139, 311

Barnes, G., \& Leka, K. D. 2008, ApJ, 688, L107

Canfield, R.C., de La Beaujardiere, J.F., Fan, Y., et al. 1993, ApJ, 411, 362

Canfield, R.C., Hudson, H.S., \& McKenzie, D.E. 1999, Geophys. Res. Lett., 26, 627

Chae, J., Moon, Y.J., \& Park, Y.D. 2004, Sol. Phys., 223, 39

Chumak, O., Zhang, H., \& Gou, J. 2004, Astronomical and Astrophysical Transactions, 23, 525

Cui, Y.M., Li, R., Zhang, L.Y., He, Y.L., \& Wang, H.N. 2006, Sol. Phys., 237, 45

De Rosa, M. L., Schrijver, C. J., Barnes, G., et al. 2009, ApJ, 696, 1780

Falconer, D. A., Moore, R. L., \& Gary, G. A. 2003, J. Geophys. Res.(Space Phys.), 108, 1380

Falconer, D.A., Moore, R.L., \& Gary, G.A. 2006, ApJ, 644, 1258 
Fan, Y. L., Wang, H. N., He, H., \& Zhu, X. S. 2011, ApJ, 737, 39

Gao, Y., Zhao, J., \& Zhang, H. 2012, ApJ, 761, L9

Gao, Y., Zhao, J., \& Zhang, H. 2014, Sol. Phys., 289, 493

Gilchrist, S.A., Wheatland, M.S., \& Leka, K.D. 2012, Sol. Phys., 276, 133

Guo, J., Zhang, H., Chumak, O. V., \& Liu, Y. 2006, Sol. Phys., 237, 25

Hagino, M., \& Sakurai, T. 2004, PASJ, 56, 831

Hagyard, M., Low, B.C., \& Tandberg-Hanssen, E. 1981, Sol. Phys., 73, 257

Hagyard, M.J., Teuber, D., West, E.A., \& Smith, J.B. 1984, Sol. Phys., 91, 115

Hudson, H.S. 1991, Sol. Phys., 133, 357

Jing, J., Song, H., Abramenko, V., Tan, C., \& Wang, H. 2006, ApJ, 644, 1273

Jing, J., Tan, C., Yuan, Y., et al. 2010, ApJ, 713, 440

Jiang, C., Feng, X., Wu, S. T., \& Hu, Q. 2012, ApJ, 759, 85

Judge, P.G., Habbal, S., \& Landi, E. 2013, Sol. Phys., 161

Leka, K. D. 1999, Sol. Phys., 188, 21

Leka, K. D., \& Barnes, G. 2003, ApJ, 595, 1277

Leka, K.D., Barnes, G., Crouch, A.D., et al. 2009, Sol. Phys., 260, 83

Li, H., Sakurai, T., Ichimoto, K., \& UeNo, S. 2000a, PASJ, 52, 465

Low, B.C. 1982, Sol. Phys., 77, 43

Kane, S. R., McTiernan, J. M., \& Hurley, K. 2005, å, 433, 1133 
McIntosh, P. S. 1990, Sol. Phys., 125, 251

Metcalf, T. R., Litao, J., McClymont, A. N., Canfield, R. C., \& Uitenbroek, H. 1995, ApJ, 439, 474

Molodensky, M.M. 1974, Sol. Phys., 39, 393

Moon, Y.J., Choe, G. S., Yun, H. S., Park, Y. D., \& Mickey, D. L. 2002, ApJ, 568, 422

Parnell, C.E., DeForest, C.E., Hagenaar, H.J., et al. 2009, ApJ, 698, 75

Press, W. H., Teukolsky, S. A., Vetterling, W. T., \& Flannery, B. P. 1992, Cambridge: University Press, c1992, 2nd ed.

Priest, E.R. 1984, Solar Magnetohydrodynamics, D. Reidel Publishing Company

Priest, E.R., \& Forbes, T.G. 2002, A\&A Rev., 10, 313

Schrijver, C.J., De Rosa, M.L., Title, A. M., \& Metcalf, T.R. 2005, ApJ, 628, 501

Schrijver, C. J., De Rosa, M. L., Metcalf, T., et al. 2008, ApJ, 675, 1637

Seehafer, N. 1978, Sol. Phys., 58, 215

Seehafer, N. 1990, Sol. Phys., 125, 219

Song, Q., Huang, G., \& Tan, B. 2012, ApJ, 750, 160

Sturrock, P.A. 1967, in P. A. Sturrock (ed.), Plasma Astrophysics, Academic Press, P.168

Taylor, J.B. 1974, Phys. Rev. Letters, 33, 1139

Taylor, J.B. 1976, in D. E. Evans (ed.), Pulsed High Beta Plasmas, Pergamon Press, Oxford, p.59

Tian, L., Wang, J., \& Wu, D. 2002, Sol. Phys., 209, 375 
Turmon, M., Jones, H. P., Malanushenko, O. V., \& Pap, J. M. 2010, Sol. Phys., 262, 277

Van Doorsselaere, T., Nakariakov, V.M., \& Verwichte, E. 2007, A\&A, 473, 959

Wang, H., Ewell, M. W., Jr., Zirin, H., \& Ai, G. 1994, ApJ, 424, 436

Wang, J., Shi, Z., Wang, H., \& Lue, Y. 1996, ApJ, 456, 861

Wheatland, M.S. 2000, ApJ, 532, 1209

Wiegelmann, T. 2004, Sol. Phys., 219, 87

Wu, S. T., Wang, A. H., Liu, Y., \& Hoeksema, J. T. 2006, ApJ, 652, 800

Wang, A. H., Wu, S. T., Liu, Y., \& Hathaway, D. 2008, ApJ, 674, L57

Woltjer, L. 1958, Proceedings of the National Academy of Science, 44, 489

Yang, X., Zhang, H.Q., Gao, Y., Guo, J. \& Lin, G.H., 2012, Sol. Phys.,

Zhang, H. 2001, ApJ, 557, L71

Zhang, H., Zi, G., Yan, X., Li, W., \& Liu, Y. 1994, ApJ, 423, 828

Zirin, H., \& Wang, H. 1993, Sol. Phys., 144, 37 
Table 1: Definitions of the physical parameters used in the paper.

\begin{tabular}{clcc}
\hline \hline Index & Description & Variable & Formula \\
\hline 1 & Free Magnetic energy $^{1}$ & $E_{1 \text { free }}$ & $\frac{1}{8 \pi} \int\left(B^{2}-B^{p^{2}}\right) d V$ \\
2 & Free Magnetic energy $^{2}$ & $E_{2 f r e e}$ & $\frac{1}{8 \pi} \int\left(\mathbf{B}-\mathbf{B}^{p}\right)^{2} d V$ \\
3 & Free Magnetic energy $^{3}$ & $E_{3 f r e e}$ & $\frac{1}{2}\left(E_{1 \text { free }}+E_{2 \text { free }}\right)$ \\
4 & Relative energy error & $r_{u n}$ & $\frac{1}{4 \pi} \int\left|\mathbf{B}^{p} \cdot\left(\mathbf{B}^{p}-\mathbf{B}\right)\right| d V / E_{3 f r e e}$ \\
5 & Total unsigned flux & $\Phi$ & $\int|\mathbf{B}| \cdot \mathbf{d} \mathbf{S}$ \\
6 & Flux imbalance & $r_{i m}$ & $\left|\int B_{z} d s\right| / \int\left|B_{z}\right| d s$ \\
7 & Total unsigned current & $I_{t}$ & $\frac{1}{4 \pi} \int\left|\partial B_{y} / \partial x-\partial B_{x} / \partial y\right| d s$ \\
8 & Proxy of free energy & $E_{p e}$ & $\frac{1}{8 \pi} \int\left(\mathbf{B}-\mathbf{B}^{p}\right)^{2} d s$ \\
9 & Averaged twist measure & $\alpha_{a v}{ }^{\mathrm{a}}$ & $\sum J_{z}(x, y) \operatorname{sign}\left[B_{z}(x, y)\right] / \sum\left|B_{z}(x, y)\right|$ \\
10 & Flare index & $F I$ & $\left(100 \sum_{\tau} I_{X}+10 \sum_{\tau} I_{M}+1 \sum_{\tau} I_{C}+0.1 \sum_{\tau} I_{B}\right) / I_{C 1.0}$ \\
\hline
\end{tabular}

${ }^{a}$ It is calculated following that of Hagino \& Sakurai (2004). 
Table 2. Information of 61 chosen ARs

\begin{tabular}{|c|c|c|c|c|c|c|c|c|}
\hline NO. & $\mathrm{AR}$ & Date & Frames & $\begin{array}{l}\text { Maxi }^{\mathrm{a}} \\
\text { Flare }\end{array}$ & Loc & $\begin{array}{l}\operatorname{Begin}^{\mathrm{b}} \\
(\mathrm{UT})\end{array}$ & $\begin{array}{l}\text { End } \\
(\mathrm{UT})\end{array}$ & $\begin{array}{l}\text { Volume } \\
\left(\mathrm{Mm}^{3}\right)\end{array}$ \\
\hline 1 & 11072 & $10 / 05 / 20-26^{\mathrm{c}}$ & 144 & B6.5 & S16W32 & $15: 46(25)$ & $15: 55$ & $183 \times 183 \times 174$ \\
\hline 2 & 11084 & $10 / 07 / 02$ & 24 & & $\ldots$ & $\ldots$ & . & $183 \times 183 \times 174$ \\
\hline 3 & 11093 & $10 / 08 / 07$ & 5 & M1.0 & N12E31 & $18: 24$ & $18: 47$ & $305 \times 192 \times 174$ \\
\hline 4 & 11112 & $10 / 10 / 16$ & 24 & M2.9 & S19W29 & 19:07 & $19: 15$ & $174 \times 157 \times 174$ \\
\hline 5 & 11158 & $11 / 02 / 12-16$ & 12 & $\mathrm{X} 2.2$ & $\mathrm{~S} 21 \mathrm{~W} 21$ & 01:44 (15) & 02:06 & $218 \times 218 \times 174$ \\
\hline 6 & 11164 & $11 / 03 / 01-06$ & 146 & $\mathrm{C} 8.6$ & N20W39 & $14: 41(06)$ & $14: 47$ & $261 \times 235 \times 174$ \\
\hline 7 & 11165 & $11 / 03 /($ & 48 &. & $\cdots$ & $\cdots$ & . & $0 \times 174$ \\
\hline 8 & 11166 & $11 / 03 / 06-10$ & 120 & $\mathrm{X} 2.2$ & N08W05 & $23: 13$ & $23: 29$ & $218 \times 218 \times 174$ \\
\hline 9 & 11169 & $11 / 03 / 09-14$ & 133 & $\mathrm{C} 5.0$ & N21E22 & $20: 56(09)$ & $21: 25$ & $261 \times 148 \times 174$ \\
\hline 10 & 11283 & $11 / 09 / 03-08$ & 120 & $\mathrm{X} 2.1$ & N14W18 & $22: 1$ & $22: 24$ & $218 \times 182 \times 174$ \\
\hline 11 & 11287 & $11 / 09 / 08-11$ & 9 & $\cdots$ & $\ldots$ & $\ldots$ & $\cdots$ & $261 \times 235 \times 174$ \\
\hline 12 & 11289 & $11 / 09 / 09-11$ & 53 & $\mathrm{C} 1.7$ & N22E26 & $17: 21(10)$ & $17: 31$ & $261 \times 157 \times 174$ \\
\hline 13 & 11302 & $11 / 09 / 25-28$ & 71 & M4.0 & N13E35 & 05:06 (26) & $05: 13$ & $261 \times 183 \times 174$ \\
\hline 14 & 11305 & $11 / 09 / 28-30$ & 54 & $\mathrm{~N}$ & N08E06 & $18: 55(30)$ & $19: 15$ & $253 \times 130 \times 174$ \\
\hline 15 & 11339 & $11 / 11 / 05-11$ & 36 & M1.8 & N21E34 & $20: 31(06)$ & $20: 54$ & $261 \times 235 \times 174$ \\
\hline 16 & 11346 & $11 / 11 / 15$ & 24 & M1.9 & S18E26 & $12: 30$ & $12: 50$ & $331 \times 209 \times 174$ \\
\hline 17 & 11354 & $11 / 11 / 20-24$ & 118 & C6.1 & S15E38 & $16: 35(20)$ & $17: 07$ & $218 \times 131 \times 174$ \\
\hline 18 & 11358 & $11 / 11 / 25-29$ & 100 & $C_{1} 1$ & N19E21 & $12: 02(26)$ & $12: 06$ & $261 \times 174 \times 174$ \\
\hline 19 & 11361 & $11 / 11 / 27-04$ & 157 & C 3.2 & N19E26 & 18:22 (28) & $18: 39$ & $218 \times 131 \times 174$ \\
\hline 20 & 11362 & 11/11/30-06 & 154 & C4.8 & N08W20 & 16:05 (04) & $16: 22$ & $261 \times 131 \times 174$ \\
\hline
\end{tabular}


Table 2-Continued

\begin{tabular}{|c|c|c|c|c|c|c|c|c|}
\hline NO. & $\mathrm{AR}$ & Date & Frames & $\begin{array}{l}\text { Maxi }^{\mathrm{a}} \\
\text { Flare }\end{array}$ & Loc & $\begin{array}{l}\text { Begin }^{b} \\
(\mathrm{UT})\end{array}$ & $\begin{array}{l}\text { End } \\
(\mathrm{UT})\end{array}$ & $\begin{array}{l}\text { Volume } \\
\left(\mathrm{Mm}^{3}\right)\end{array}$ \\
\hline 21 & 11363 & $11 / 12 / 02-07$ & 140 & C6.9 & S20W09 & $23: 20(05)$ & $23: 34$ & $261 \times 174 \times 174$ \\
\hline 22 & 11374 & $11 / 12 / 10-13$ & 75 & C1.6 & S17E43 & $23: 57(10)$ & 00:07 & $100 \times 131 \times 174$ \\
\hline 23 & 11375 & $11 / 12 / 10-13$ & 80 & $\mathrm{C} 1.2$ & N09E38 & 06:24 (10) & $06: 43$ & $200 \times 78 \times 174$ \\
\hline 24 & 11381 & $11 / 12 / 19-24$ & 139 & C5.4 & S19W18 & 01:56 (22) & $02: 20$ & $218 \times 113 \times 174$ \\
\hline 25 & 11386 & $11 / 12 / 27-31$ & 120 & $\mathrm{C} 8.9$ & S17E32 & $04: 11(27)$ & 04:31 & $218 \times 113 \times 174$ \\
\hline 26 & 11387 & $11 / 12 / 25-26$ & 48 & M4.0 & S22W26 & $18: 11(25)$ & $18: 20$ & $158 \times 87 \times 174$ \\
\hline 27 & 11389 & $11 / 12 / 31$ & 24 & M2.4 & S25E44 & $13: 09$ & $13: 19$ & $287 \times 244 \times 174$ \\
\hline 28 & 11428 & $12 / 03 / 05-11$ & 140 & $\mathrm{C} 7.2$ & S18W03 & 02:49 (08) & $02: 56$ & $200 \times 131 \times 174$ \\
\hline 29 & 11429 & $12 / 03 / 06-10$ & 114 & $\mathrm{X} 5.4$ & N17E27 & 00:02 (07) & 00:40 & $218 \times 166 \times 174$ \\
\hline 30 & 11430 & $12 / 12 / 05-11$ & 128 & $\mathrm{X} 1.3$ & N22E12 & 01:05 (07) & $01: 27$ & $183 \times 131 \times 174$ \\
\hline 31 & 11471 & $12 / 05 / 01-07$ & 135 & M1.5 & S19W44 & 14:03 (07) & $14: 52$ & $348 \times 166 \times 174$ \\
\hline 32 & 11476 & $12 / 05 / 08-13$ & 132 & M4.7 & N13E31 & $12: 21(07)$ & $12: 36$ & $305 \times 200 \times 174$ \\
\hline 33 & 11494 & $12 / 06 / 04-08$ & 111 & M2.1 & S19W05 & $19: 54(06)$ & $20: 13$ & $218 \times 122 \times 174$ \\
\hline 34 & 11504 & $12 / 06 / 11-17$ & 124 & M4.7 & S16E18 & $11: 29(13)$ & $14: 31$ & $200 \times 122 \times 174$ \\
\hline 35 & 11505 & $12 / 06 / 11-17$ & 111 & $\mathrm{C} 1.9$ & S09E10 & 00:32 (13) & $00: 38$ & $131 \times 87 \times 174$ \\
\hline 36 & 11512 & $12 / 06 / 26-01$ & 141 & $\mathrm{C} 4.2$ & S16E09 & 04:45 (28) & $04: 55$ & $218 \times 131 \times 174$ \\
\hline 37 & 11513 & $12 / 06 / 29-04$ & 133 & $\mathrm{C} 1.9$ & N14E04 & 19:11 (01) & $19: 21$ & $174 \times 174 \times 174$ \\
\hline 38 & 11515 & $12 / 06 / 30-06$ & 153 & M6.1 & S20W32 & 11:39 (05) & $11: 49$ & $261 \times 166 \times 174$ \\
\hline 39 & 11520 & $12 / 07 / 11-14$ & 75 & $\mathrm{X} 1.4$ & S19E05 & $15: 373(12)$ & $17: 30$ & $218 \times 218 \times 174$ \\
\hline 40 & 11532 & $12 / 07 / 29$ & 7 & M2.3 & S22E40 & $06: 15$ & $06: 29$ & $331 \times 218 \times 174$ \\
\hline
\end{tabular}


Table 2-Continued

\begin{tabular}{|c|c|c|c|c|c|c|c|c|}
\hline NO. & $\mathrm{AR}$ & Date & Trat & $\begin{array}{l}\text { Maxi }^{\mathrm{a}} \\
\text { Flare }\end{array}$ & Loc & $\begin{array}{l}\text { Begin }^{\mathrm{b}} \\
(\mathrm{UT})\end{array}$ & $\begin{array}{l}\text { End } \\
(\mathrm{UT})\end{array}$ & $\begin{array}{l}\text { Volume } \\
\left(\mathrm{Mm}^{3}\right)\end{array}$ \\
\hline 41 & 11535 & $12 / 08 / 01-06$ & 125 & $\mathrm{C} 3.0$ & N18E14 & $21: 20(03)$ & $21: 31$ & $218 \times 1$ \\
\hline 42 & 11542 & $12 / 08 / 09-14$ & 107 & $\mathrm{C} 4.2$ & S16E36 & 04:07 (10) & $04: 45$ & $227 \times 157 \times 174$ \\
\hline 43 & 11543 & $12 / 08 / 10-16$ & 137 & C3.6 & N19W36 & $12: 41(16)$ & $13: 48$ & $227 \times 174 \times 174$ \\
\hline 44 & 1554 & $12 / 08 / 24-26$ & 74 & $\mathrm{C} 1.7$ & 16E12 & $02: 24(25)$ & $02: 55$ & $200 \times 105 \times 174$ \\
\hline 45 & 11560 & $12 / 08 / 30-04$ & 125 & C5.5 & N07W16 & 18:00 (02) & $18: 15$ & $174 \times 131 \times 174$ \\
\hline 46 & 11564 & $12 / 09 / 03-08$ & 122 & M1.4 & S15W36 & $17: 35(08)$ & $18: 20$ & $348 \times 218 \times 174$ \\
\hline 47 & 11565 & $12 / 09 / 04-07$ & 65 & $\mathrm{C} 2.3$ & N10E20 & $07: 0$ & $07: 20$ & 116 \\
\hline 48 & 1 & $12 / 09 / 13$ & 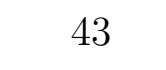 & $C$ & 5 & 2. & 57 & 16 \\
\hline 49 & 11613 & $12 / 11 / 13-19$ & 129 & M2.8 & S22E33 & 20:50 (13) & $20: 57$ & $218 \times 174 \times 174$ \\
\hline 50 & 11618 & $12 / 11 / 18-24$ & 132 & M3.5 & N06E07 & $15: 10(21)$ & $15: 38$ & $261 \times 122 \times 174$ \\
\hline 51 & 11620 & $12 / 11 / 24-27$ & 96 & trt & S14W41 & $21: 05(27)$ & 30 & $218 \times 113 \times 174$ \\
\hline 52 & 11635 & $12 / 12 / 21-28$ & 160 & C4.1 & N11W14 & $12: 58(25)$ & 13:07 & $253 \times 183 \times 174$ \\
\hline 53 & 11652 & $13 / 01 / 08-15$ & 138 & M1.7 & N19W21 & 08:35 (13) & $08: 40$ & $305 \times 200 \times 174$ \\
\hline 54 & 11654 & $13 / 01 / 11-17$ & 104 & $\mathrm{~N}$ & N06E39 & 14:51 (11) & $15: 24$ & $305 \times 209 \times 174$ \\
\hline 55 & 11665 & $13 / 02 / 01-0$ & 120 & $\mathrm{C}$ & N10W04 & $17: 29$ & $18: 32$ & $208 \times 105 \times 174$ \\
\hline 56 & 11675 & $13 / 02 / 16-20$ & 64 & M1.9 & N12E22 & $15: 45(17)$ & $15: 52$ & $121 \times 46 \times 116$ \\
\hline 57 & 11692 & $13 / 03 / 13-18$ & 130 & M1.1 & N11E12 & 05:46 (15) & $08: 35$ & $203 \times 151 \times 174$ \\
\hline 58 & 11695 & $13 / 03 / 16-18$ & 65 & $\mathrm{C} 1.0$ & N08W04 & 14 & $14: 56$ & $244 \times 9$ \\
\hline 59 & 11696 & $13 / 03 / 12-16$ & 108 & $\mathrm{C} 2.2$ & N07W17 & 10:39 (15) & $10: 46$ & $244 \times 96 \times 174$ \\
\hline 60 & 11718 & $13 / 04 / 06-12$ & 153 & M3.3 & N19W42 & 19:52 (12) & $20: 46$ & $218 \times 122 \times 174$ \\
\hline
\end{tabular}


Table 2-Continued

\begin{tabular}{lcccccccc}
\hline \hline & & & & & & & \\
NO. AR & Date & Frames & Maxi $^{\mathrm{a}}$ & Loc & Begin $^{\mathrm{b}}$ & End & Volume \\
& & & Flare & & $(\mathrm{UT})$ & $(\mathrm{UT})$ & $\left(\mathrm{Mm}^{3}\right)$ \\
\hline & & & & & & & & \\
61 & 11719 & $13 / 04 / 08-14$ & 138 & M6.5 & N09E12 & $06: 55(11)$ & $07: 29$ & $305 \times 244 \times 174$ \\
\hline
\end{tabular}

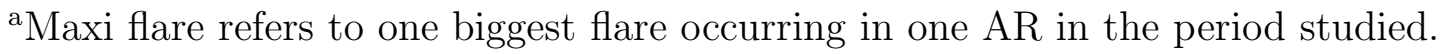

b The number in parentheses is the date of the flare burst.

' It denotes the time.

Table 3: Index $\alpha$ of the frequency-size distributions of FME, FI and magnetic flux.

\begin{tabular}{ccccc}
\hline \hline & $F I_{2-\text { day }}$ & $F I_{3-\text { day }}$ & $E_{3 \text { free }}$ & $\Phi$ \\
\hline$F I \geqslant 0$ & $1.6 \pm 0.6(0.90)^{\mathrm{a}}$ & $1.4 \pm 0.6(0.99)$ & $2.0 \pm 0.4(0.98)$ & $1.8 \pm 0.5(0.99)$ \\
$F I_{1-\text { day }} \geqslant 1$ & $1.5 \pm 0.5(0.95)$ & $1.4 \pm 0.4(0.99)$ & $1.9 \pm 0.4(0.98)$ & $1.4 \pm 0.5(0.99)$ \\
$F I_{1-\text { day }} \geqslant 10$ & $1.5 \pm 0.5(0.95)$ & $1.3 \pm 0.4(0.99)$ & $1.7 \pm 0.4(0.98)$ & $\ldots$ b \\
$F I_{2-\text { day }} \geqslant 1$ & $1.5 \pm 0.6(0.90)$ & $1.4 \pm 0.4(0.99)$ & $1.9 \pm 0.4(0.98)$ & $1.5 \pm 0.6(0.99)$ \\
$F I_{2-\text { day }} \geqslant 10$ & $1.5 \pm 0.6(0.90)$ & $1.5 \pm 0.4(0.99)$ & $1.8 \pm 0.4(0.95)$ & $0.8 \pm 0.5(0.99)$ \\
$F I_{2-\text { day }} \geqslant 50$ & $1.5 \pm 0.9(0.75)$ & $1.4 \pm 0.6(0.99)$ & $1.5 \pm 0.5(0.98)$ & $1.2 \pm 0.6(0.99)$ \\
$F I_{3-\text { day }} \geqslant 1$ & $1.5 \pm 0.6(0.90)$ & $1.4 \pm 0.4(0.99)$ & $1.9 \pm 0.4(0.98)$ & $1.9 \pm 0.6(0.99)$ \\
$F I_{3-\text { day }} \geqslant 10$ & $1.5 \pm 0.6(0.90)$ & $1.4 \pm 0.4(0.99)$ & $1.8 \pm 0.4(0.98)$ & $1.0 \pm 0.5(0.99)$ \\
$F I_{3-\text { day }} \geqslant 50$ & $1.5 \pm 0.6(0.90)$ & $1.4 \pm 0.6(0.99)$ & $1.6 \pm 0.5(0.98)$ & $1.3 \pm 0.5(0.98)$ \\
\hline
\end{tabular}

${ }^{a}$ The number in parentheses is the significance level of $\chi^{2}$-test.

${ }^{b}$ The value is not shown as it is much less than the error. Likewise, when the threshold is set as $F I \geqslant 50$ in the time window $\tau=1$ day, the values of $\alpha$ do not make sense and are not shown. 
Table 4. Flare productivity for $E_{3 \text { free }}=5 \times 10^{32}$ ergs or $\sim \Phi=3 \times 10^{22} \mathrm{Mx}$ under different threshold of $F I$ and different time window of $\tau$-days.

\begin{tabular}{|c|c|c|c|c|c|c|}
\hline \multirow[b]{2}{*}{$F I$} & \multicolumn{2}{|c|}{$\tau=1$-day } & \multicolumn{2}{|c|}{$\tau=2$-days } & \multicolumn{2}{|c|}{$\tau=3$-days } \\
\hline & $E_{3 \text { free }}$ & $\Phi$ & $E_{3 \text { free }}$ & $\Phi$ & $E_{3 f r e e}$ & $\Phi$ \\
\hline$\geq 1(\mathrm{C} 1.0)$ & 0.95 & 0.85 & 0.98 & 0.93 & 1.00 & 0.95 \\
\hline$\geq 10(\mathrm{M} 1.0)$ & 0.70 & 0.45 & 0.90 & 0.70 & 0.95 & 0.80 \\
\hline$\geq 50(\mathrm{M} 5.0)$ & 0.28 & 0.15 & 0.45 & 0.40 & 0.50 & 0.45 \\
\hline$\geq 100(\mathrm{X} 1.0)$ & 0.20 & 0.10 & 0.30 & 0.15 & 0.32 & 0.32 \\
\hline
\end{tabular}

Table 5: Correlations of the magnetic measures with $F I / \Phi$ for all the data $(F I \geq 0)$.

\begin{tabular}{ccccc}
\hline \hline & $F I_{1-\text { day }}$ & $F I_{2-\text { day }}$ & $F I_{3-\text { day }}$ & $\Phi$ \\
\hline$E_{3 \text { free }}$ & $0.53(100 \%)^{\mathrm{a}}$ & $0.57(100 \%)$ & $0.58(100 \%)$ & $0.91(100 \%)$ \\
$\Phi$ & $0.50(100 \%)$ & $0.55(100 \%)$ & $0.57(100 \%)$ & $1.00(100 \%)$ \\
$I_{t}$ & $0.53(100 \%)$ & $0.59(100 \%)$ & $0.60(100 \%)$ & $0.98(100 \%)$ \\
$E_{p e}$ & $0.59(100 \%)$ & $0.64(100 \%)$ & $0.65(100 \%)$ & $0.96(100 \%)$ \\
$\alpha_{a v}$ & $0.42(100 \%)$ & $0.42(100 \%)$ & $0.42(100 \%)$ & $0.23(100 \%)$ \\
\hline
\end{tabular}

${ }^{a}$ The number in parentheses is the Spearman rank correlation's confidence level. 

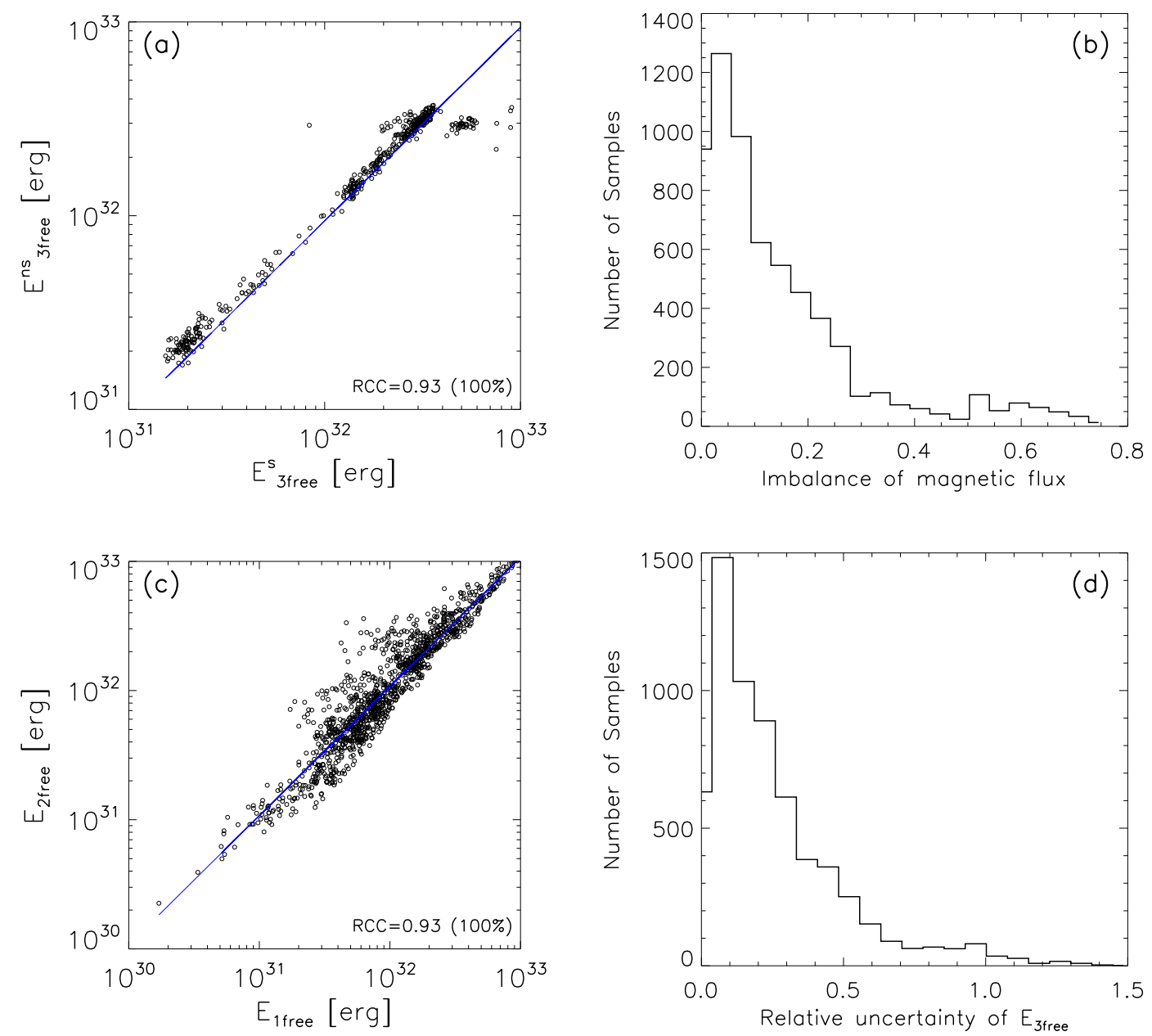

Fig. 1. - Assessment of the quantities related to the uncertainty of the determined FMEs. Panel (a) corresponds to a data sample of NOAA AR 11158 alone, while panels (b-d) correspond to the entire data sample. (a) $E_{3 f r e e}^{s}$ vs. $E_{3 f r e e}^{n s}$, obtained from the testing vector magnetograms being smoothed and not smoothed, respectively. (b) Histogram of the relative imbalance of magnetic flux. (c) $E_{1 \text { free }}$ vs. $E_{2 \text { free }}$ (d) Histogram of the relative uncertainty of $E_{3 f r e e}$. The blue lines are linear fittings to the data. 

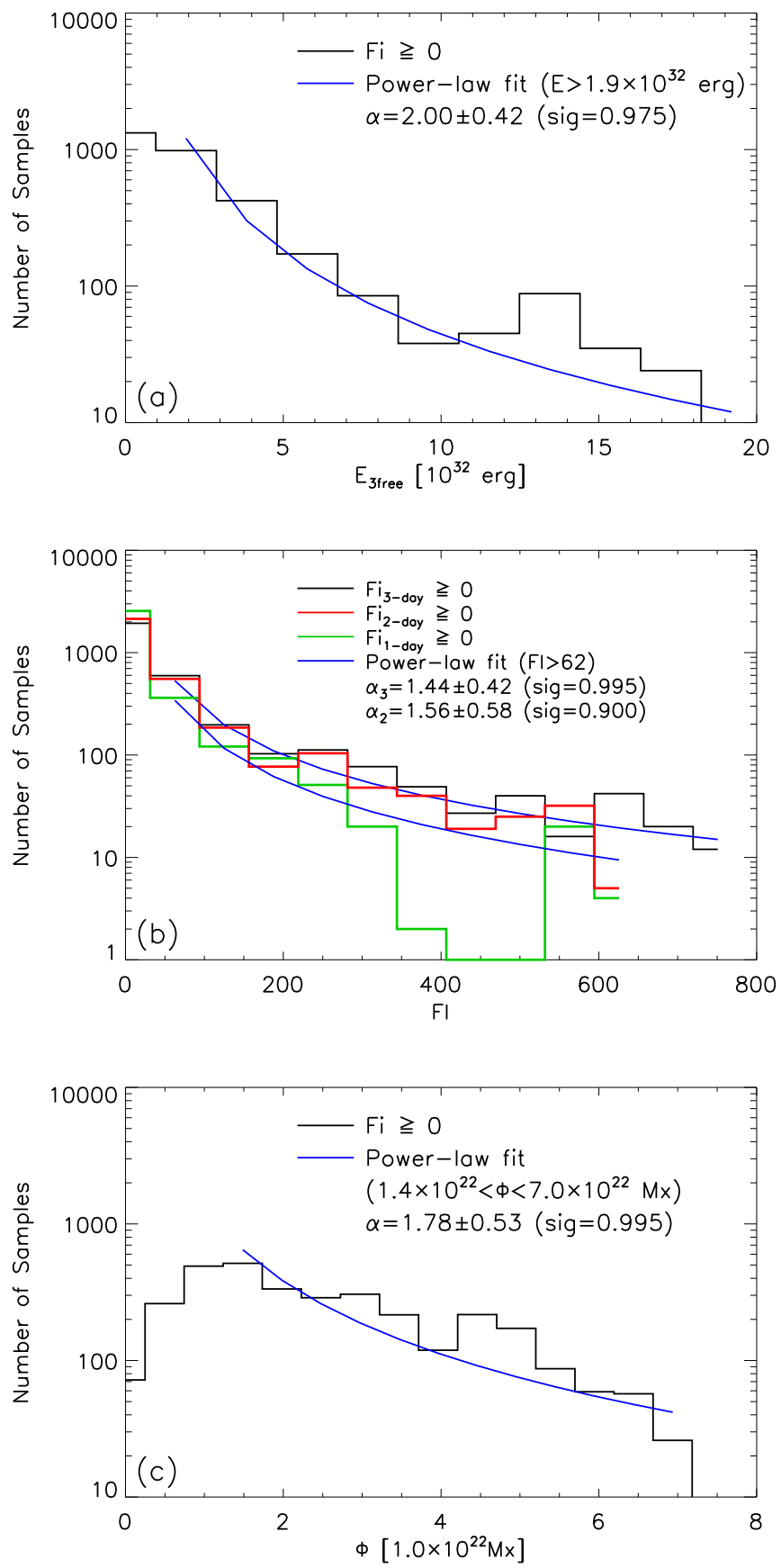

Fig. 2.- Histograms of FME (a), FI (b) and magnetic flux (c) of all the data (FI $\geq 0)$. The blue lines are the nonlinear fittings to the histograms (black or red) created in the $\chi^{2}$-test. In panel (b), the FI for $\tau=1$-day window was not fitted due to insufficient data when $F I>350$ (the sample size $N<5$ ). Likewise, the data in panel (c) was not fitted when $\Phi>7 \times 10^{22}$ Mx. The lower-cutoff and the significance level are marked in parentheses. 

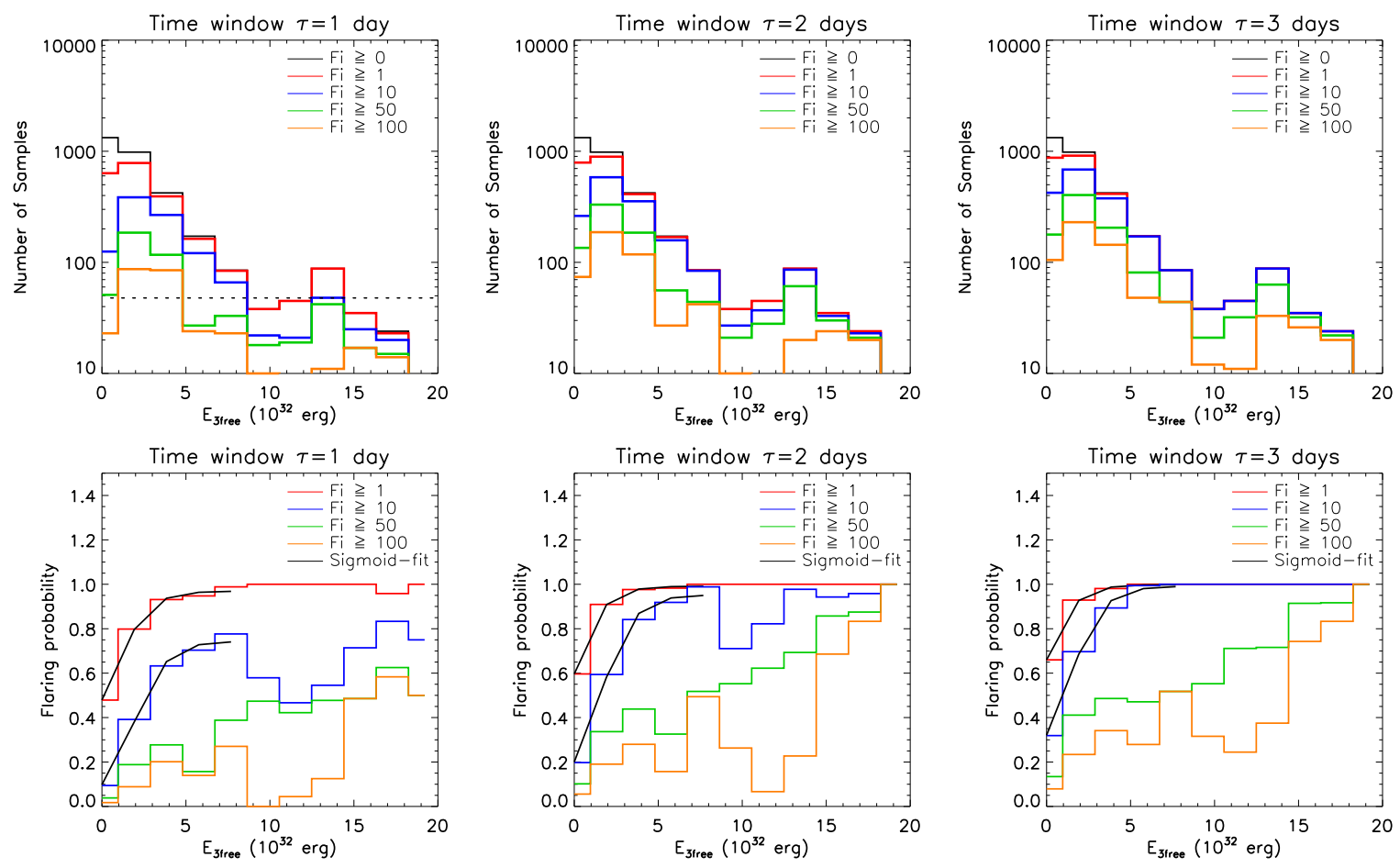

Fig. 3.- Histograms of $E_{3 \text { free }}$ (top panels) and flare productivity $P$ (bottom panels) predicted with the measurements in the time windows of $\tau=1-3$ days for different thresholds of FI (indicated by black, red, blue, green and yellow colors). The solid black lines in the bottom panels are the sigmoid function fittings to the flare productivity with the sample size $N>50$. 

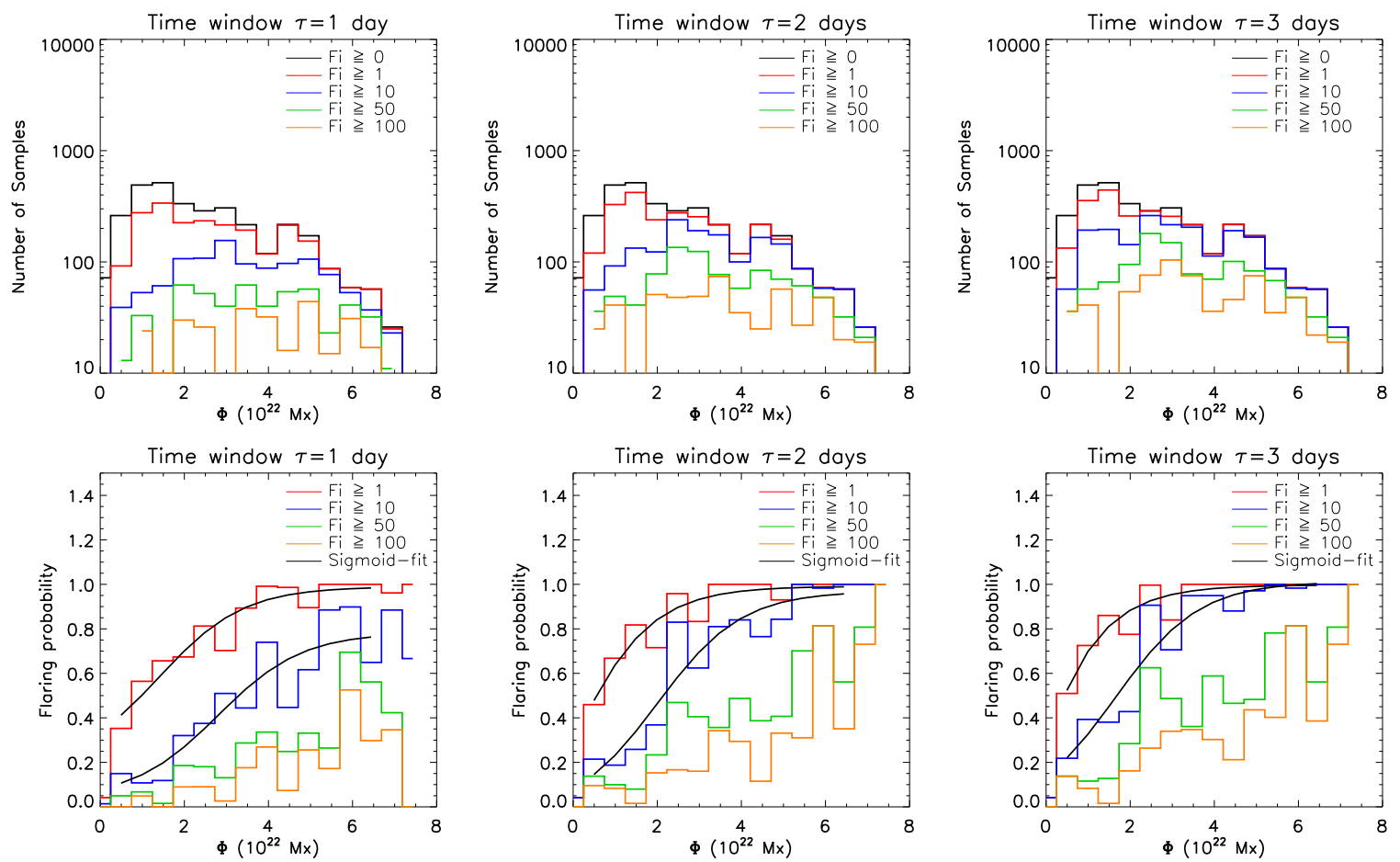

Fig. 4. - Same as Figure 3, but for the measure of $\Phi$. 

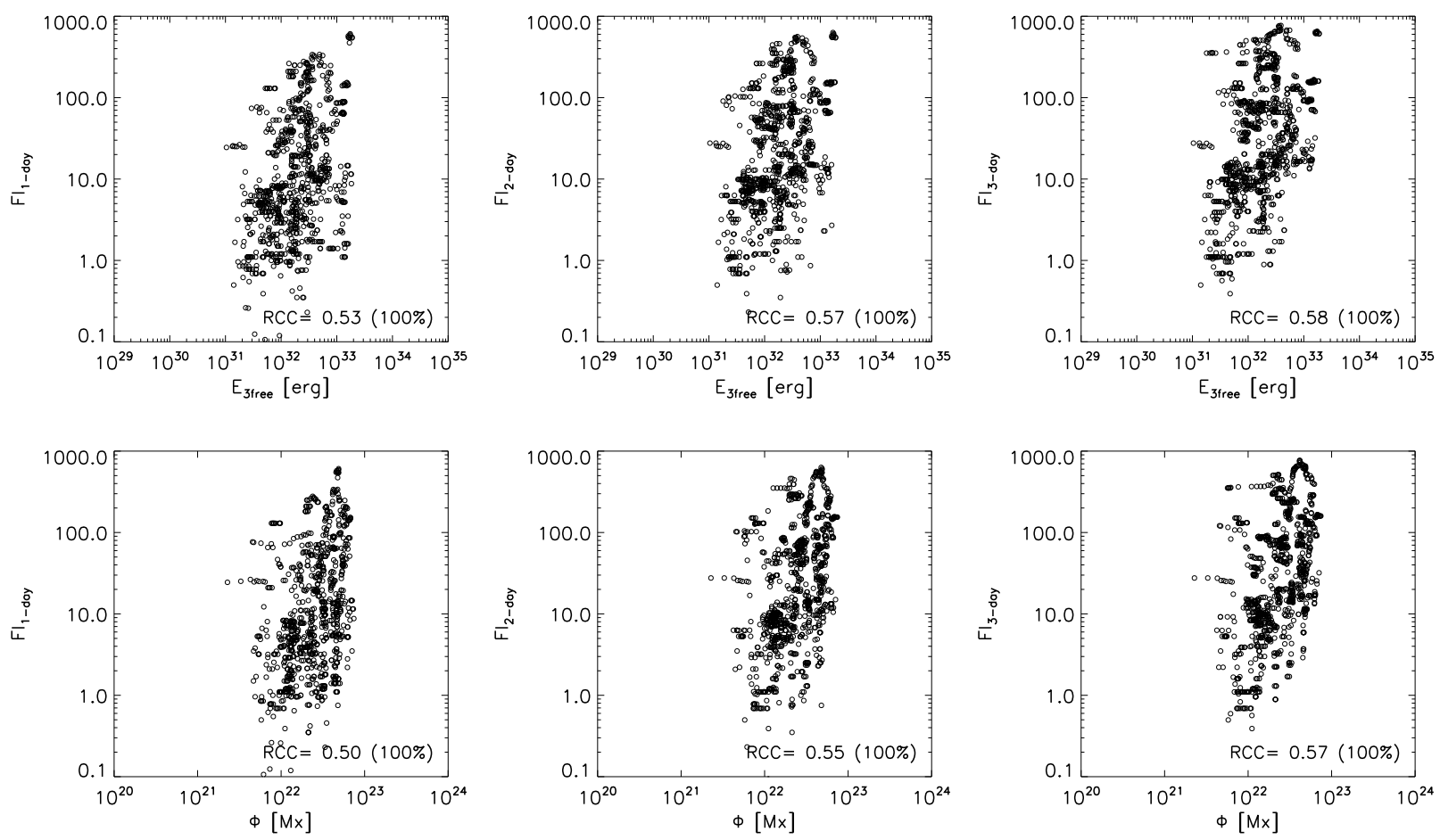

Fig. 5.- Scatter plots of the FI versus the magnetic measures $E_{3 \text { free }}$ (top panels) and $\Phi$ (bottom panels). Shown from left to right are the panels in the time windows of $\tau=1-3$ days, respectively. The number in the bottom right corner of each panel is the Spearman rank-correlation coefficient and that in parentheses is the correlation's confidence level in percent. 

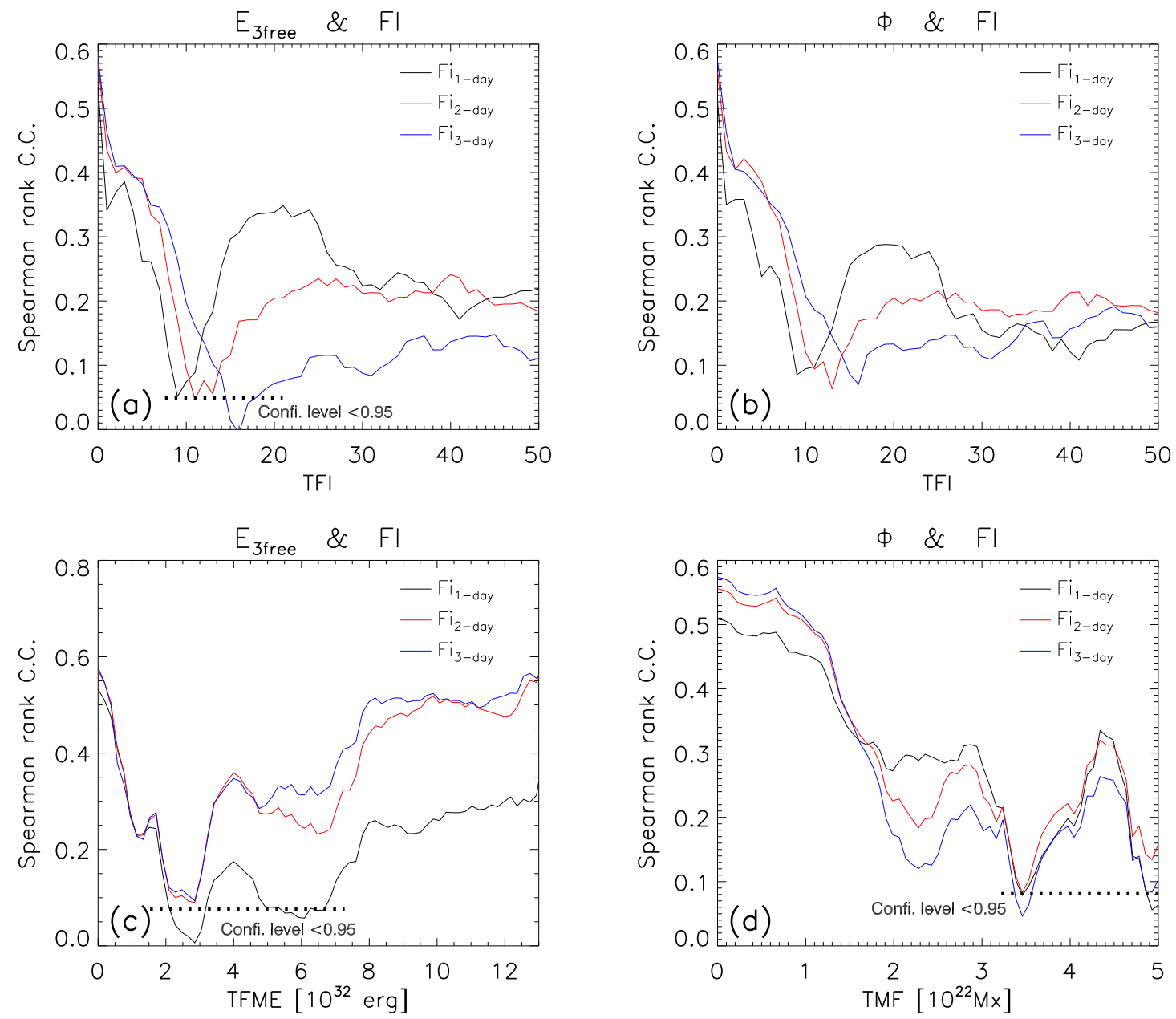

Fig. 6.- Correlation varying with the threshold of the parameters, FI, FME and $\Phi$. The RCC between $E_{3 \text { free }}$ and $F I$ versus $T F I$ is shown in (a), and that versus $T F M E$ in (c). The RCC between $\Phi$ and $F I$ versus $T F I$ is shown in (b), and that versus $T M F$ in (d). The curves in the time windows of $\tau=1-3$ days are illustrated by black, red and blue colors, respectively. The data below the dotted-lines have confidence level $<0.95$. 

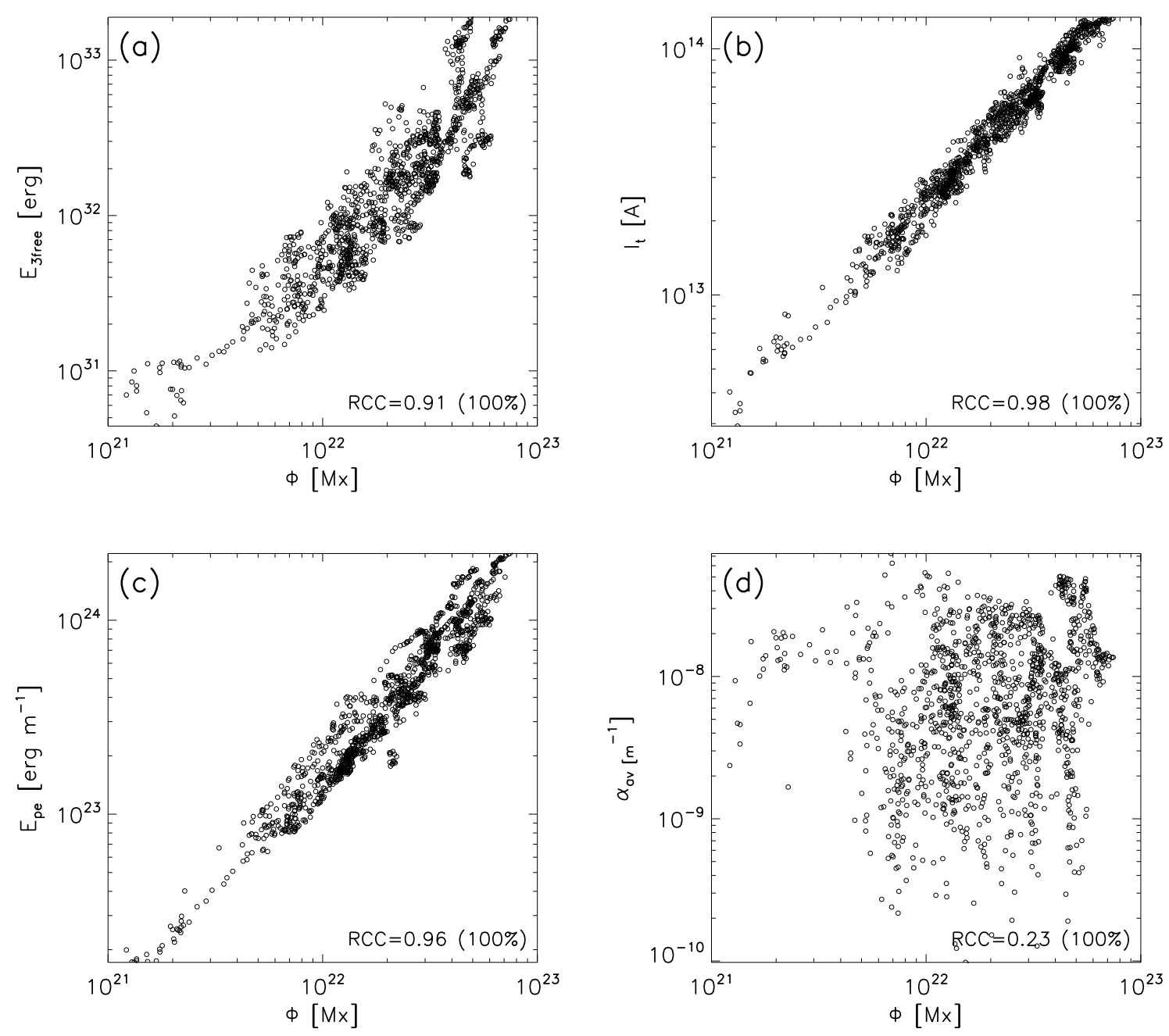

Fig. 7.- Scatter plots of $\Phi$ vs. $E_{3 f r e e}(\mathrm{a}), I_{t}(\mathrm{~b}), E_{p e}(\mathrm{c})$ and $\alpha_{a v}(\mathrm{~d})$. The number in the bottom right corner of each panel is the Spearman rank-correlation coefficient and that in parentheses is the correlation's confidence level in percentage. 